\title{
The Analysis of the Aromatic Amino Acids Interaction with Poly-N-Vinylpyrrolidone using UV and IR Spectroscopy
}

\author{
Oksana A. Pakhomova*, Nadezhda Ya. Mokshina and Dmitry A. Minakov \\ Yelets State Bunin University, Yelets, Russian Federation; monblan.pro@yandex.ru
}

\begin{abstract}
Quantitative characteristics of interphase distribution of aromatic amino acids were obtained under identical conditions; a general framework for the analysis was developed including preliminary extraction of analytes from aqueous salt solutions using an extractant based on Poly-N-Vinyl Pyrrolidone. Application of ammonium sulfate as a salting-out agent proved to be feasible. Spectral analysis methods allowed establishing the structure of complexes formed during amino acids extraction. The structure of distributed complexes was determined and the mechanisms of the interaction of amino acids with the polymer were proposed based on the data concerning characteristic absorption band displacement in the IR spectra of the complexes. Conditions for almost complete extraction of phenylalanine, tyrosine and tryptophan from aqueous salt solutions were optimized. The method for extraction-spectrophotometric determination of aromatic amino acids in aqueous solution was developed. The studied extraction systems are applicable for almost complete extraction of phenylalanine, tyrosine and tryptophan from aqueous solutions during the analysis of various genesis media, including food products and pharmaceuticals.
\end{abstract}

Keywords: Absorption Spectra, Aromatic Amino Acids, Extraction, Poly-N-Vinyl Pyrrolidone, UV and IR Spectroscopy

\section{Introduction}

Preparation of amino acid pharmaceuticals is one of the priority trends of the scientific and technological development; it is promising and is of high demand on an industrial scale. Complexes of amino acids with vitamins, carbohydrates and lipids are widely used for treatment of diseases of the major part of organ systems. The effectiveness of such medicinal products is based on the anabolic and anti-oxidant effect, stimulation of energy production and regeneration ${ }^{1-3}$. Application of free amino acids, unlike protein, does not require energy consumption for digestion during absorption. The level of free amino acids in blood increases faster than when using protein or dextrans and the pharmaceuticals technology $y^{4}$.

Invention of new and improvement and development of known methods of amino acids separation and concentration, allows extending the capabilities of analytical methods in the field of the pharmaceuticals technology ${ }^{5}$.
One of the major issues during production of amino acids resides in complete extraction, concentration and separation. The search for new, environmentally friendly and effective extraction systems for concentration of essential amino acids is associated with the development of express methods for their selective determination in various environments. Liquid extraction and its promising trend - "green" extraction, which presupposes application of non-toxic water-soluble high molecular weight compounds - are still compatible with modern requirements to the methods of concentration and separation of biologically active substances ${ }^{6}$.

The fundamental limitation of traditional extraction systems relates to the fact that substances with significant hydration energy usually are poorly extracted into the organic phase, which limits application of extraction processes ${ }^{7}$. In addition, the organic solvent-water system cannot be used for work with biologically active substances due to their collapsibility. This explains the

* Author for correspondence 
interest in two-phase water systems based on watersoluble polymers safe for biological objects that had previously been proposed and used to resolve a number of crucial biotechnological tasks.

Instrumental and titrimetric determinations exclude re-extraction stage and allow for selective determination of homologues and isomers, forecasting quantitative characteristics of interphase distribution of organic compounds.

Tyrosine extraction was studied in the "octyl alcohol - water" system with the distribution ratio of 5.5 .103. The study includes data on extraction of aromatic amino acids by means of alcohols-homologues, as well as on distribution of amino acids in the "high alcohols - saturated aqueous salt solution" system. A linear correlation between the distribution ratio and the structure of hydrocarbon radical of alcohols was determined. Initial concentrations of phenylalanine, tyrosine and tryptophan were $0.1-1 \mathrm{mg} / \mathrm{cm}^{3}$, the distribution ratio was $\mathrm{D}<13$. The extractants used do not ensure sufficiently complete extraction of amino acids from aqueous solutions.

We studied the extraction of $\alpha$-amino acids with aromatic substituents - synthetic water-soluble polymers and proposed the extraction systems for removal and separation of phenylalanine, tyrosine and tryptophan by means of water-soluble carbo- and heterochain polymers of the poly-N-vinyl amides and polyethylene glycol range. To improve the sorption characteristics with regard to amino acids, the nonionic sorbent Stirosorb is modified with polyvinylpyrrolidone.

The water-soluble poly-N-vinyl amides are applied for effective extraction of varying amino acids from aqueous media. The study determined the influence of the structure and molecular weight of Poly-N-Vinyl Pyrrolidone, Poly-N-Vinyl Caprolactam and Poly-NVinyl-N-Methylacetamide on the rate of biologically active substances recovery ${ }^{8}$.

\section{Related Data}

Liquid extraction and its promising trend - "green" extraction, which presupposes application of non-toxic water-soluble high molecular weight compounds - are still compatible with the modern requirements to the methods of concentration and separation of biologically active substances. Over the last years, the chemistry of poly$\mathrm{N}$-vinyl amides has been intensively developing and has integrated into many fields of science, including medicine and biotechnology. Water solubility, biocompatibility, nontoxicity, thermosensitivity in aqueous solutions and high complexable capacity - all these properties of poly$\mathrm{N}$-vinyl amides determine future areas of their practical application ${ }^{9,10}$.

It is known that UV and IR spectroscopy methods were successfully applied to determine composition and structures of the complexes and associates in diverse systems, including biologically active substances ${ }^{11,12}$. Herewith, the mechanism of interaction of amino acids with extractants, water-soluble polymers in particular, before and after extraction are still underexplored.

The purpose of this work is the spectroscopic study of the interaction mechanism of individual aromatic amino acids and their mixtures with Poly-N-Vinyl Pyrrolidone to justify the choice of extraction systems.

The study was conducted in the following basic steps: - Synthesis of carbon-chain polymers based on N-vinyl amides and $\mathrm{N}$-vinilazolami different by molecular weights in the range of $10.103-200.103$ using the method of free-radical homo- and copolymerization in isopropyl alcohol; determination of their intrinsic viscosity;

- Study of extraction of aromatic amino acids using polymers and copolymers; obtaining quantitative characteristics of extraction under identical conditions; development of a general scheme for analysis;

- Spectroscopic study (UV and IR spectroscopy) of the interaction mechanism of individual aromatic amino acids and their mixtures with Poly-N-Vinyl Pyrrolidone to justify the choice of extraction systems.

- Development of methods for concentration and extraction-spectrophotometric determination of aromatic amino acids in aqueous solution.

The subjects of study are aromatic amino acids phenylalanine and tryptophan - differing by the structure of hydrocarbon radical, the number of carboxyl and amine groups. They are widely used as food additives, therapeutic agents and intermediate substances of organic synthesis; in modern medicine, aromatic amino acids are used for diagnosis, treatment and prevention of many diseases ${ }^{13}$.

It is known that most of $\alpha$-amino acids are characterized by a wide spectrum of biological activity. For example, phenylalanine is an original substance in the synthesis of antibodies, hormones, enzymes and other biologically active compounds. Tryptophan normalizes the imbalance in the system of central monoamines and 
neuroactive amino acids of brain (taurine, 5-hydroxy-Ltryptophan, dopamine and serotonin) in the setting of subchorionic phenobarbital toxicity ${ }^{14}$.

Extraction is a common method of removal, separation and concentration of amino acids. Specific features of extraction include flexibility, the determination potential in a wide range of concentrations, compatibility with different concentrate analysis methods and rapidity. Amino acids extraction schemes proven in processing media of microbiologic manufacturing were developed based on the physical-chemical studies of extraction processes $^{15}$.

The ion exchange ${ }^{16}$, membrane ${ }^{17}$ and solid phase ${ }^{18}$ extraction is applied for concentration of amino acids.

Sulfo-derivatives dissociating in a wide $\mathrm{pH}$ range are the most effective ion exchange extractants. For instance, the solution of dinonylnaphthalene sulfonic acid as the ion exchanger is used for extraction of phenylalanine and tryptophan from microbiological media. The method for extraction of amino acids from the cell culture fluid was developed based on the ion exchange extraction using dinonylnaphthalene sulfonic acid. The main factor affecting the dependence of the selectivity of extraction on the exchange capacity of ion exchanger is the nature of the functional groups in the ion-exchanger state (liquid or polymer).

Sulfonic acids as extractants are more selective with regard to amino acids than sulphocationite ${ }^{19}$.

The co-extraction effect, which decreases with addition of a polar solvent into the extractant phase, is characteristic of amino acids extraction from multicomponent solutions. The separation factor for leucine, isoleucine, valine and lysine is equal to 1 in a wide $\mathrm{pH}$ range. Analogous dependence is noticeable during separation of other aliphatic and aromatic amino acids ${ }^{20}$.

Membrane extraction of amino acids was studied using the mathematical models of a hollow sphere (stationary diffusion) and the advancing front (nonstationary transfer). This process is the limiting stage of membrane extraction.

The method of direct microcalorimetry was used to study solid-phase extraction process of glycine, glutamic acid, tyrosine by anion exchanger $\mathrm{AB}-17-8$ from aqueous solutions at $25^{\circ} \mathrm{C}$. Amino acids in solutions at $\mathrm{pH} \approx 11$ are in the form of anions. The interaction of anions of amino acids with functional groups of the anion exchanger was studied. During determination of amino acids at the concentration of $0.05-0.1 \mathrm{~mol} / \mathrm{dm}^{3}$ standard deviation does not exceed $2 \%^{21}$.

A method was developed for determination of glycine and glutamic acid in aqueous media based on solid-phase extraction using carboxyl cation exchanger $\mathrm{CB}-2$ and CB-4 in $\mathrm{Cu}^{2+}$-form. Maximal adsorption of amino acids from aqueous solutions was determined at various $\mathrm{pH}$ values.

The advantage of solid-phase extraction consists in the possibility of separation of substances with similar properties, a low detection limit, rapidity. Limitations are associated with a low degree of amino acids extraction from aqueous media. Extraction of trace quantities of amino acids was studied by means of supercritical fluid extraction using $\mathrm{CO}_{2}$ or mixtures of $\mathrm{CO}_{2}$ with alkali (modifier). Optimum conditions allows for up to $48 \%$ recovery of some $\alpha$-amino acids from medicinal herbs.

A common method for concentration of many organic compounds, including amino acids is the liquid extraction method ${ }^{22}$. Extractants are hydrophilic and hydrophobic organic compounds of different classes. In addition to straightforward extraction activity, hydrophilic compounds differ by the fact that their determination is possible by means of physicochemical, e.g., electrochemical methods, in respective concentrates.

\section{Method}

We prepared aqueous-salt solutions of phenylalanine and tryptophan with the concentration of 0.35 and $0.075 \mathrm{mg} / \mathrm{ml}$, accordingly (the salting-out agent was the saturated ammonium sulfate solution). Poly-N-Vinyl Pyrrolidone (PVP) with the molecular weight of 3.500 and the concentration of aqueous solution of $0.12 \mathrm{~g} / \mathrm{ml}$ was used as extractant. Radical polymerization of the $\mathrm{N}$-Vinyl Pyrrolidone monomer in isopropanol solution was conducted to obtain polymer at $65^{\circ} \mathrm{C}$ and with the concentration of dinitrileazobisisobutyric acid (the initiating agent) equal to $10^{-2} \mathrm{~mol} / \mathrm{l}$. Average viscosity molecular weight of polymer was calculated using the Mark-Kuhn-Houwink equation, taking into account the experimentally found values of the intrinsic viscosity in ethanol at $25^{\circ} \mathrm{C}$.

The extraction of tryptophan and phenylalanine from aqueous solutions with Poly-N-Vinyl Pyrrolidone-3500 was studied.

PVP-3500 is a white amorphous polymer readily soluble in water, chloroform, ethanol and almost insoluble in aromatic hydrocarbons and ketones. 


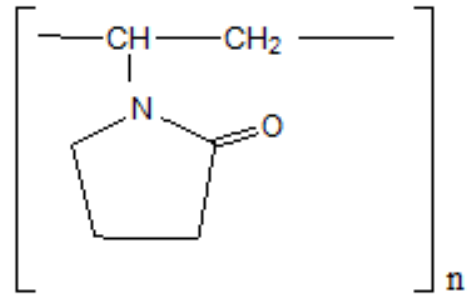

Poly-N-Vinyl Pyrrolidone

It interacts with many low- and high-molecular compounds in aqueous solutions, is capable of complex formation with various organic and inorganic substances. This property extends practical application of the polymer in a variety of industries.

Unlimited miscibility of the polymer with water at temperatures below phase separation is possible only due to the hydrophobic interaction that stabilizes the structure of water and makes it possible to form a singlephase solution. The polymer can be dissolved in ketones and unsaturated hydrocarbons.

Due to partial or complete solubility of hydrophilic solvents in water, a prerequisite for the extraction is saturation of the aqueous solution with electrolyte reducing the solubility of the substance to be distributed in water and providing delamination of the system.

The liquid two-phase system is obtained based on the aqueous solution of polyelectrolyte and the saltingout agent. Herewith, one of the phases is saturated with polymer and the second - with salt ${ }^{23}$.

The salting-out effect of electrolytes with reference to organic compounds can be explained by reduction of unbound water volume in aqueous solution. The saltingout agent facilitates the exchange of water molecules from the immediate environment of the extracted ion for molecules of the organic solvent.

The effectiveness of the salting-out agent depends on the nature of electrolyte and the properties of the distributed substance. Salts have impact on the dielectric conductivity of the medium, the ionic strength of the solution; improve the quantitative extraction characteristics (the distribution and concentration ratios, the $\mathrm{R}$ recovery rate).

Widespread use of $\mathrm{Li}+, \mathrm{Na}+, \mathrm{K}+$ and $\mathrm{NH} 4+$ salts as salting-out agents is determined by their good water solubility.

The influence of competing ions grows with the increasing solubility of salt, resulting in the decrease of the distributed substance hydration and the salting out action strengthening.
It was found that the recovery rate of phenylalanine and tryptophan is significantly affected by the volume ratio of the aqueous and organic phases due to the reciprocal solubility of phases ${ }^{24}$.

After extraction recovery and separation of organic compounds, an analysis of the aqueous concentrate is to be conducted using various physicochemical methods ${ }^{25}$ as well as capillary electrophoresis.

Capillary electrophoresis is based on electrokinetic phenomena - electromigration of ions and other charged particles and electroosmosis. These phenomena occur in solutions placed in an electric field, predominantly of high voltage. If the solution is in a thin capillary, e.g. in a quartz one, the electric field applied along the capillary induces the motion of charged particles and passive fluid flow, whereby the sample is split into individual components, as electromigration parameters are specific for each type of charged particles. At the same time, such perturbation factors as diffusion, sorption, convection, gravity, etc. are substantially weakened in the capillary, due to which the peak efficiency of separation is achieved.

The analysis was performed using the "Kapel-105M" capillary electrophoresis system with high voltage positive polarity (inner diameter of the capillary: 50 micron; total length: $75 \mathrm{~cm}$ ). The analysis performed at the recommended $40^{\circ} \mathrm{C}$ showed a peak value interfering determination of nicotinic acid. Therefore, the temperature was raised to $50^{\circ} \mathrm{C}$ without changing the pressure and detection wavelength. To obtain reliable results, the absorption spectrum of nicotinic acid in the sample was recorded simultaneously with the electrophoretogram.

The optimum composition of buffer solution and concentration of the micelle-forming agent was selected through the optimization of conditions of the electrophoretic analysis of nicotinic acid in the pharmaceutical composition. The study determined the impact of different concentrations of the micelleforming agent on separating ability of the borate buffer and conditions of nicotinic acid extraction from vitamins (composition and $\mathrm{pH}$ of the extractant, time and temperature). The modeled mixtures of excipients commonly used in the manufacture of solid pharmaceutical forms (cornstarch, glucose, lactose, citric acid, tartrazine dye) were analyzed at optimized conditions. Electropherograms were made to eliminate the influence of the buffer solution and the extractant.

Spectrophotometry is characterized by low detection limits, varied selection of light absorption bands and is widely used to study the structure and composition of 
various compounds, for qualitative and quantitative determination of substances of different classes ${ }^{26}$.

The IR spectra of samples were recorded in the 4.000$400 \mathrm{~cm}^{-1}$ range of frequencies using the "Bruker Vertex 70 FT" IR spectrophotometer with a Fourier transformer by means of frustrated total internal reflection. The scanning conditions were: 32 scans, resolution: $4 \mathrm{~cm}^{-1}$.

The UV spectra of amino acids' solutions, polymer and aqueous phase after extraction were registered using the "Suimadzy" (Japan) device in $1 \mathrm{~cm}$ thick quartz cuvettes within the range of $190-300 \mathrm{~nm}$. The registration parameters were: the band gap $-0.5 \mathrm{~mm}$, slow mode, the recording interval being $0.5 \mathrm{~nm}$.

\section{Results}

It was found that the recovery rate of phenylalanine and tryptophan is significantly affected by the volume ratio of aqueous and organic phases due to the high reciprocal solubility of phases (Figure 1).

The dependence of tryptophan recovery rate in PVP3500 systems from the volume ratio of equilibrium phases indicates the highest system performance with the f value equal to $10: 4$

The polymer solution viscosity and the rate of delamination with salt solutions depend on the molecular weight of the polymer and its concentration in the system. The polymer concentration varied within the range of $0.05-0.15 \mathrm{~g} / \mathrm{cm}^{3}$. Preliminary studies showed that the concentration over $0.15 \mathrm{~g} / \mathrm{cm}^{3}$ results in formation of polymer associates, while the bound water is released transferring into the equilibrium aqueous-salt phase.

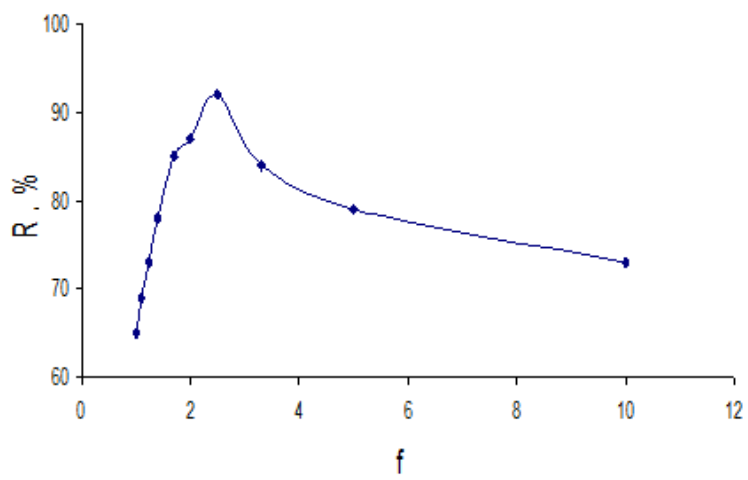

Figure 1. The tryptophan recovery rate versus the volume ratio of equilibrium phases during extraction of PVP-3500 by ammonium sulfate.
As a result, the equilibrium ratio of volume phases remains constant and the vitamins distribution ratios slightly increase as the concentration of the polymer exceeds $0.15 \mathrm{~g} / \mathrm{cm}^{3}$.

The maximum extraction characteristics of amino acids are obtained at $0.12 \mathrm{~g} / \mathrm{cm}^{3}$ concentration of PVP3500 .

Quantification of amino acids is based on their ability to absorb electromagnetic radiation in the ultraviolet spectral range.

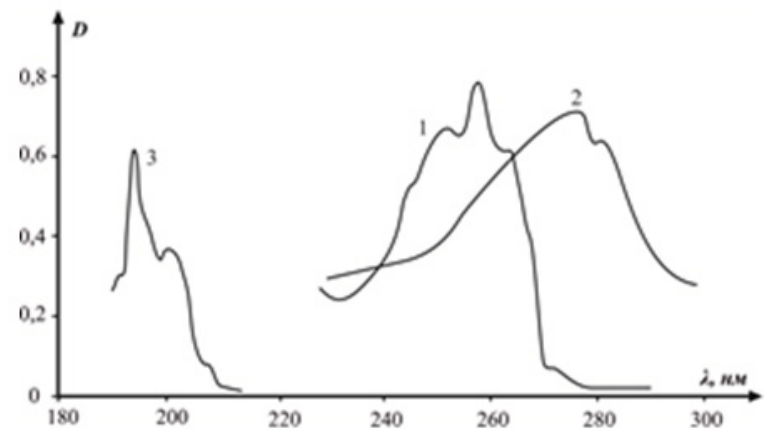

Figure 2. The UV absorption spectra of phenylalanine (1), tryptophan (2) and Poly-N-Vinyl Pyrrolidone (3) in an aqueous solution.

Figure 2 shows the spectra of absorption of phenylalanine (curve 1), tryptophan (curve 2), and Poly-N-Vinyl Pyrrolidone (curve 3) in aqueous solution. Polymer and amino acids absorption bands do not overlap; therefore, in case of extracting one of the studied amino acids, it is possible to effectively detect the presence of a certain amino acid and quantify its concentration. The equilibrium concentration of amino acids can be calculated at characteristic wavelengths: $257 \mathrm{~nm}$ for phenylalanine and 279nm for tryptophan. Presence of the salting-out agent in extraction systems also does not shift the absorption maximum and does not interfere with the determination of amino acids.

However, in case of simultaneous extraction of two amino acids, the UV range absorption spectroscopy method is ineffective for separate determination of them due to the substantial overlapping of the absorption bands of the individual amino acids (Figure 1). In addition, the UV spectra analysis does not provide information about the mechanism of the polymer interaction with amino acids. 


\section{Discussion}

To study the mechanism of $\alpha$-amino acids distribution in the phase of Poly-N-Vinyl Pyrrolidone, we recorded the absorption spectra in the infrared range of the polymer and organic phase after extraction (Figure 3). Spectral display of amino acids in the solution resides in the significantly increasing intensity of the absorption bands that are responsible, for instance, for bending vibrations of the $\mathrm{CH}_{2}$-group $\left(1.292 \mathrm{~cm}^{-1}\right.$ and $\left.1.464 \mathrm{~cm}^{-1}\right)$, deformation scissoring vibrations of the $\mathrm{CH} 2$-group $\left(1.444 \mathrm{~cm}^{-1}\right)$. It should be noted that there were no detected valence vibrations in the spectrum for the $\mathrm{NH}_{3}^{+}$group, included in the structure of amino acids and corresponding to the wave numbers 3.120-3.130 and $2.140 \mathrm{~cm}^{-1}$ (deformation vibrations of the $\mathrm{NH}^{3+}$ group correspond to the band of approx. $\left.1.478 \mathrm{~cm}^{-1}\right)$. Herewith, valence vibrations of water overlap with the absorption bands of the functional groups of amino acids within the range of $3.120-3.130 \mathrm{~cm}^{1}$. Moreover, Figure 3 illustrates slight reduction of intensity of bands in the range of $3.420 \mathrm{~cm}^{-1}$ and $3.220 \mathrm{~cm}^{-1}$ corresponding to the valence vibrations of water.

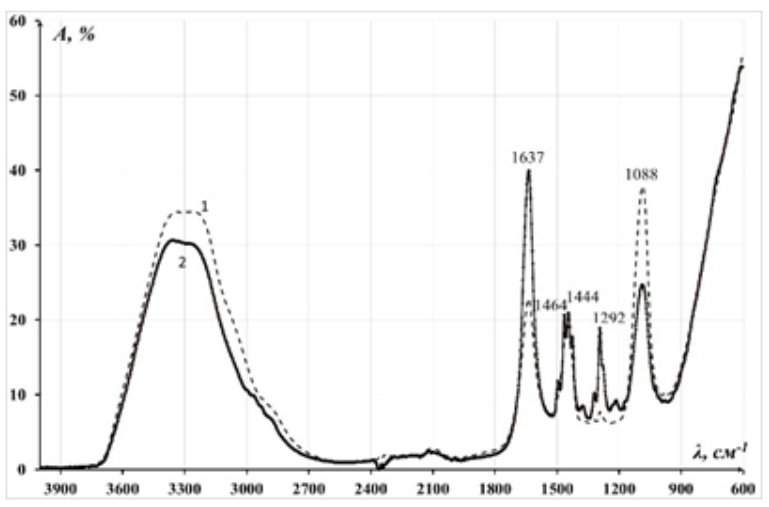

Figure 3. The IR absorption spectra of aqueous solution of Poly-N-Vinyl Pyrrolidone (1) and an organic phase containing phenylalanine and tryptophan (2).

Therefore, it can be assumed that the association of amino acids, water and the polymer in the organic phase after extraction is carried out by means of hydrogen bonds between the amino groups of amino acids and $\mathrm{CO}$-groups of Poly-N-Vinyl Pyrrolidone.

This assumption can be indirectly confirmed by the fact that the increase of absorption of solution with amino acids with a maximum of $1.637 \mathrm{~cm}^{-1}$ is registered in the spectrum. As is known, this band corresponds to the bending vibrations of water. Probably valence vibrations of CO-ionized groups, the maximum of which corresponds to the wave number $1.650 \mathrm{~cm}^{-1}$, have certain influence on this band. The intensity of this band is increased in the presence of amino acids.

Thus, these results suggest that polymer complex formation with amino acids in aqueous media occurs due to formation of $\mathrm{H}$-bonds between $>\mathrm{C}=\mathrm{O}$ groups of polymers cycle and the functional groups of amino acids with participation of water molecules. The repeating PVP unit and amino acids can interact as follows:

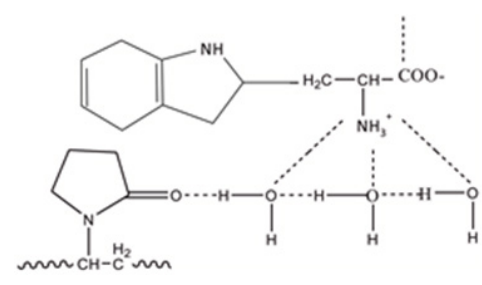

Thus, complex formation with the nitrogen atom of the polymer cycle is unlikely due to the steric factor from the part of the main chain.

Table 1. Extraction characteristics during recovery of phenylalanine and tryptophan PVP-3500 at various concentrations of amino acids. The ratio of aqueous and organic phases is $10: 4 ; \mathrm{n}=4 ; \mathrm{P}=0.95$

\begin{tabular}{ccc}
\hline $\mathrm{C}, \mathrm{mg} / \mathrm{cm}^{3}$ & $\mathrm{D}$ & $\mathrm{R}, \%$ \\
\hline \multicolumn{3}{c}{ Phenylalanine } \\
\hline 0.20 & $10 \pm 2.3$ & 80 \\
0.25 & $14 \pm 1.8$ & 85 \\
0.30 & $18 \pm 0.9$ & 88 \\
0.35 & $23 \pm 1.4$ & 90 \\
0.40 & $17 \pm 2.7$ & 87 \\
& Tryptophan & \\
0.060 & $19 \pm 2.2$ & 88 \\
0.065 & $25 \pm 3.1$ & 91 \\
0.070 & $28 \pm 1.2$ & 92 \\
0.075 & $36 \pm 2.1$ & 94 \\
0.080 & $30 \pm 2.5$ & 93 \\
\hline
\end{tabular}

The studied mechanism of complex formation in the "amino acid - PVP solution" system is proven by high extraction characteristics of the system (Table 1). The maximum recovery rate of phenylalanine at single extraction in the presence of ammonium sulfate is $90 \%$, tryptophan $-94 \%$ and tyrosine $-92 \%$.

The UV and IR spectroscopy methods were applied to determine the structure of distributed complexes. 
The study proposed the mechanisms of interaction of amino acids with the polymer. Based on displacement of characteristic absorption bands in the IR spectra at extraction of amino acids, it was found that the interaction of functional groups of amino acids and polymers occurs due to hydrogen bonds. It was found that the use of polymer with lower molecular weight leads to higher extraction characteristics; therefore, almost complete extraction of aromatic amino acids in a single extraction was achieved in the proposed systems.

\section{Conclusion}

We have studied the extraction of phenylalanine and tryptophan from aqueous salt solutions using watersoluble synthetic polymers; obtained quantitative characteristics of extraction under identical conditions; developed the general framework for the analysis; explored the impact of aqueous and organic phases' volume ratio on the degree of phenylalanine and tryptophan extraction and also studied the dependence of polymer solution viscosity and systems separation rate from molecular weight of the extractant and its concentration.

We have found out that the studied aromatic amino acids are most fully extracted using the PVP-3500 solution at the $0.12 \mathrm{~g} / \mathrm{cm}^{3}$ concentration and the equilibrium volume ratio of aqueous and organic phases equal to 10:4; optimized the conditions for almost complete extraction of phenylalanine, tyrosine and tryptophan from aqueous salt solution and developed the technique for extractionspectrophotometric determination of aromatic amino acids in aqueous solution.

Eventually, we have proposed the scheme for interaction of PVP-3500 with recoverable materials based on maximum light absorbance. The developed technique is quick (the analysis time is $30-40$ minutes), precise (the relative error does not exceed 7\%) and environmentally friendly (no toxic and noxious extractants, "green extraction"). The studied extraction systems are applicable for almost complete extraction.

We used the UV and IR spectroscopy methods to determine the structure of distributed complexes and proposed the mechanisms of interaction of amino acids with the polymer. We have found that the use of polymer with lower molecular weight leads to higher extraction characteristics; therefore, almost complete extraction of aromatic amino acids in a single extraction was achieved in the proposed systems.

The studies conducted during the reporting period allowed to formulate a common methodological approach to the extraction of aromatic amino acid by water-soluble polymers. The results are statistically reliable to verify the effectiveness of synthetic polymers application with different structures for concentration of biologically active substances by the example of phenylalanine and tryptophan.

Further research in this field with an increased number of objects of analysis and extractants is deemed prospective. In particular, the studies focusing on extraction of vitamins, their concentration and separation in complex mixtures, including amino acids, are of immediate interest. A number of synthetic copolymers should be studied as extractants and based on the obtained data; techniques for quantitative determination of amino acids and vitamins in complex mixtures by the example of real objects should be developed.

\section{Acknowledgments}

The research was carried out within of the basic part of the state task of the Ministry of Education and Science of the Russian Federation for 2015.

This work was financially supported by the Ministry of Education and Science of the Russian Federation within the framework of the state order $\# 2468$.

\section{References}

1. Kuznetsov VA. Synthesis and radical polymerization of cyclic N-vinylamides. Moscow: Moscow State Academy of Fine Chemical Technology named after M. V. Lomonosov; 1998.

2. Ivashov MN. Biological activity of compounds from herbal materials. Fundamental Research. 2013; 10(7):1482-4.

3. Anikina LV, et al. Synthesis. Anti-inflammatory and analgesic activity of naproxen amides with amino acid derivatives. Khim.-Pharm. 2002; 36(5):12-5.

4. Blinova OA. Theoretical and experimental aspects of creation of pharmaceuticals based on natural raw materials. Perm: State Educational Institution of Higher Professional Training "Perm State Pharmaceutical Academy of the Federal Agency for Health and Social Development"; 2009. p. 319.

5. Tuchinin VV. Optical biomedical diagnostics. Moscow: Fizmatlit. 2007. p. 560.

6. Tsyplukhin Yu V. Extraction of $\alpha$-amino acids with aromatic substituents synergistic water-soluble polymers. Vorone- 
zh: Voronezh State University; 2006. p. 142.

7. Kuvaeva ZI. Extraction of amino acids using sulphocationite. Chemistry and extraction technology. Moscow: Mendeleyev University of Chemical Technology of Russia; 2001. p. 319.

8. Nifantyeva TI. Extraction of humic acids in two-phase aqueous polymer systems. Analytical Chemistry Magazine. 2000; 55(10):1030-2.

9. Shatalov GV, Verezhnikov VN, Plaksitskaya TV, Kuznetsov VA, Poyarkova TN. Synthesis of copolymers N,N-dimethylaminoethyl and $\mathrm{N}$-vinyl caprolactam and features of their behavior in the processes of complex formation and flocculation. High-Molecular Compounds. B. 2006; 48(6):16.

10. Roizard D, Favre E, Teplyakov V, Fefilatiev L, Lagunstov N, Khotimsky V. Realisation et etude d'un module membranaire a base de PTMSP pour la separation de COV dilués d'effluents gazeux, Recents Progres en Genie des Procedes. 2003; 89:443-50.

11. Shatalov GV, Verezhnikov VN, Churilina EV, Kuznetsov VA, Poyarkova TN. Flocculation of latex synthetic rubber by homo- and co-polymer N-vinylcaprolactam with N-vinylimidazole. Journal of Applied Chemistry. 2003; 76(11):1890-4.

12. Chavula EV, Kusnetsov VA, Verezhnikov VN, Shatalov GV. Free radical copolymerisation of $\mathrm{N}$-vinylcaprolactam with V-vinyl-1.2.3-triazols and Properties of Related Copolymers. Polymer Science. Ser. 5. 2003; 45(2):341-6.

13. Jezova D, Makatsori A, Smriga M, Morinaga Y, Duncko R. Subchronic treatment with amino acid mixture of L-lysine and L-arginine modifies neuroendocrine activation during psychosocial stress in subjects with high trait anxiety. NutrNeurosci. 2005; 8:155-60.

14. Ladner CL, Yang J, Turner RJ, Edwards RA. Visible fluorescent detection of proteins in polyacrylamide gels without staining. Anal Biochem. 2004; 326:13-20.

15. Paiva AP. Solvent extraction associated with activation analysis: A helpful tool. J. Radio-analytical and Nuclear Chemistry. 2004; 319(1):135-41.

16. Soldatov VS. The structure and properties of extractants based on dinonylnaphthalene sulfonic acid. Chemistry and extraction technology. 2001; 1(5):19-38.

17. Fan S, Jia Q, Song N, Su R, Liao W. Synergistic extraction study of indium from chloride medium by mixtures of sec-nonylphenoxy acetic acid and trialkyl amine. Sep Purific Technol. 2010; 75:76-80.

18. Formanovskiy AA, Pletnev IV, Smirnov SV. Ionic liquids New solvents for extraction and analysis. Journal of Analytical Chemistry. 2003; 58(7):710-1.

19. Gavrilyuk IV. Extraction of phenylalanine and tryptophan using solutions of dinonylnaphthalene sulfonic acid. Minsk: IFOH. 2006. 20.

20. Latin NN, Staseva ON, Banashek VM. Natural antioxidants and valuable components of $\mathrm{CO}_{2}$-extracts. 3rd International Conference "Extraction of Organic Compounds". Voronezh. 2005. p. 113.

21. Askalepova OI. Determination of flavonoid compounds in a $\mathrm{CO}_{2}$-extracts of herbal raw material. 2nd International Symposium. Separation and concentration in analytical chemistry and radiochemistry. Krasnodar. 2005; 128.

22. Korenman YI, Pakhomov OA. Extraction separation of tyrosine and glycine. Modern High Technol. 2007; 5:16-8.

23. Pakhomova OA. New extraction system for determining a-amino acid in aqueous media. Saratov: Saratov State University; 2007. p. 148.

24. Korenman YI, Chibisova TV, Pakhomova OA. Distribution coefficients for novocaine in aliphatic alcohol (C3-C9)-solvotropicreagentsalting-out reagent-water systems. Russian J Phys Chem. 2013; 7:1218-21.

25. Khripkov MV, Peregoudov YuS, Amelin ZhS. Thermochemical solid phase extraction of amino acids from aqueous solutions. 3rd International Conference "Extraction of Organic Compounds". Voronezh. 2005. 45.

26. Kharitonov YuYa. Analytical Chemistry (analytics). In 2 books. Book 1. General theoretical framework. Qualitative analysis. Moscow: Vysshaya Shkola; 2001. p. 615. 